\title{
Food Effects Assessment on Oral Pharmacokinetics of Rosuvastatin in Wild Mice
}

\author{
Zoobia Irum ${ }^{1}$, Abdul Aleem², Nasreen Ramzan ${ }^{3}$, Inaam Ul Haq ${ }^{4}$, Usman Abid $^{3 *}$, Shiza Murad ${ }^{3}$ \\ ${ }^{1}$ Department of Pharmacology, University of Health Sciences Lahore, Pakistan \\ ${ }^{2}$ Department of Pharmacy, the Islamia University of Bahawalpur, Pakistan \\ ${ }^{3}$ Department of Pharmaceutics, Bahauddin Zakariya University, Pakistan \\ ${ }^{4}$ Department of Pharmaceutical chemistry, Bahauddin Zakariya University, Pakistan
}

DOI: $10.36348 /$ sjmps.2019.v05i11.010

| Received: 06.11.2019 | Accepted: 19.11.2019| Published: 28.11.2019

*Corresponding author: Usman Abid

\section{Abstract}

Rosuvastatin is regularly recommended for the treatment of hypercholesterolemia and applies its impact through focused collection in the liver. Current dosing rules show no inclination for sustained or fasted rosuvastatin organization. In this investigation, lower plasma level was noted in mice with nourishment 2 hours after an oral rosuvastatin portion, while liver fixation was unaffected. We presume that taking an oral portion with nourishment rather than on an unfilled stomach, don't fundamentally influence the cholesterol-bringing down limit of rosuvastatin. Since a typical unfavorable occasion noted with statin treatment is muscle torment/harm related with high coursing statin levels, our discoveries can possibly fill in as a novel and basic system for relieving statin myopathy hazard.

Keywords: rosuvastatin, oral pharmacokinetics, food effect, hepatic uptake transport, mice.

Copyright @ 2019: This is an open-access article distributed under the terms of the Creative Commons Attribution license which permits unrestricted use, distribution, and reproduction in any medium for non-commercial use (NonCommercial, or CC-BY-NC) provided the original author and source are credited.

\section{INTRODUCTION}

Statins like rosuvastatin, atorvastatin, simvastatin and so forth are the most normally recommended class of meds for the treatment of hypercholesterolemia [1]. Most statins are directed in their dynamic, hydroxy-corrosive structure; anyway simvastatin and lovastatin are managed as lactones star drugs [2]. Clinical factors including dynamic liver and renal illness can modify statin pharmacokinetics. Notwithstanding attending infection, different components that may impact statin pharmacokinetics incorporate hereditary qualities, ethnicity, age, sex, and nourishment consumption [3]. Rosuvastatin is a manufactured and one of the most powerful statins and has shown better cholesterol bringing down capacities when thought about than different statins available [4]. Current dosing rules for rosuvastatin demonstrate no inclination for nourished or fasted organization, however ongoing pharmacokinetic information recommend that rosuvastatin fundamental introduction is altogether diminished when managed with nourishment [5].

It was reasoned that this nourishment impact was likely a consequence of diminished intestinal ingestion of rosuvastatin. Be that as it may, lower fundamental presentation could likewise be clarified by expanded hepatic leeway of rosuvastatin. Along these lines, the goals for the present work were to decide the impact of simultaneously controlled nourishment on rosuvastatin pharmacokinetics and to explore the impact of related nourishment organization with rosuvastatin portion on the lipid profiles of statin patients. A potential nourishment impact is inspected inside mice. Estimate of this examination is that the plasma rosuvastatin will be lower and liver rosuvastatin level will be higher in a sustained state. For testing this theory, explore was executed as an in vivo pharmacokinetic study in wild mice.

\section{METHODOLOGY}

\section{Mouse Pharmacokinetic Study}

An in vivo pharmacokinetic study was performed in two gatherings (nourished and fasted) of wild mice (Pharmaceutics lab, mice are 9-10 weeks old; weight between 24-26g, 5 for every gathering). The mice were housed in a temperature-controlled condition where they got standard murine chow and water not obligatory. 5-6 hours before tranquilize dosing, all nourishment was expelled from the fasting mouse gathering and the bedding was changed to guarantee that there was no lingering nourishment in the confines. The bedding was likewise changed in the enclosures of the fed mice. All mice were dosed $10 \mathrm{mg} / \mathrm{kg}$ 
rosuvastatin in phosphate cradled saline by oral gavage. Around $28 \mu \mathrm{L}$ of blood was gathered preceding rosuvastatin portion ( 0 time point), and at $7.5,15,30$, and $60 \mathrm{~min}$ after medication dosing. All blood was gathered utilizing a heparinized pipet after saphenous vein cut. Following 2 hours of medication dosing, the mice were euthanized by isoflurane and the rest of the blood was gathered into EDTA-containing tubes by means of cardiovascular cut. All blood tests were centrifuged at $4-4{ }^{\circ} \mathrm{C}, 2000 \mathrm{rcf}$ for $10-12 \mathrm{~min}$ to isolate plasma and cell parts. Plasma was aliquoted into relating cryovials and put away at $-80{ }^{\circ} \mathrm{C}$. Livers from all mice were extracted after death, washed in phosphate-cradled saline, smudged, and gauged; liver examples were streak solidified in fluid nitrogen and put away at $-80^{\circ} \mathrm{C}$.

\section{Assurance of Statin Concentrations}

Mouse plasma and liver rosuvastatin focuses were resolved. In a word, two arrangements of standard bend esteems were readied. The principal set was utilized when breaking down plasma tests and was readied utilizing clear mouse plasma and rosuvastatincalcium salt. The second arrangement of standard bend esteems was utilized when examining liver examples and was readied utilizing homogenized, wild-type, untreated liver examples and rosuvastatin calcium salt. Liver examples and gauges were homogenized in $0.05 \%$ formic corrosive in water. Plasma tests and liver homogenate tests $(5 \mu \mathrm{L}$ of each) were accelerated utilizing $20 \mu \mathrm{L}$ acetonitrile containing inward standard (rosuvastatin-d6) and centrifuged for $20 \mathrm{~min}$ at 14000 rpm at $4{ }^{\circ} \mathrm{C}$. The supernatant from each example was then weakened $1: 2$ in $0.05 \%$ formic corrosive in water and examination was done.

\section{Pharmacokinetics and Statistical Analysis}

Pharmacokinetic information from the fed and fasted mouse bunches were thought about utilizing the Mann-Whitney test. Liver-to-plasma proportions were determined by isolating the tissue rosuvastatin fixation by the plasma focus at the last examining time point. Mean plasma rosuvastatin fixations at 2 hours, liver rosuvastatin focuses, and liver-to-plasma proportions were looked at among bolstered and fasted mouse bunches utilizing the Mann-Whitney test. Factual investigation was performed utilizing GraphPad Prism 6.

\section{RESULTS}

Taking rosuvastatin with nourishment has been appeared to bring about lower sedate plasma levels when contrasted with those accomplished from a fasted organization [5]. This might be expected to either diminished intestinal retention or expanded hepatic freedom of rosuvastatin within the sight of nourishment. To additionally examine a potential nourishment impact, we played out a pharmacokinetic study whereby $10 \mathrm{mg} / \mathrm{kg}$ of rosuvastatin was regulated by means of oral gavage to sustained and fasted wildtype mice. Past information demonstrated that the mean plasma AUC0- $\infty$ was roughly $127.6 \mathrm{ng} * \mathrm{~h} / \mathrm{mL}$ with a standard deviation of 23.2 [6]. Information from $n=4$ encouraged mice and $n=5$ fasted mice were dissected.

Practically identical to prior reports, we saw the oral ingestion of rosuvastatin as very quick [6]. The most noteworthy rosuvastatin plasma fixations were seen at the first run through point for both nourished and fasted mice (Figure-1). Half-existence of rosuvastatin was not altogether unique among bolstered and fasted mice (Table-1). Investigation of mean

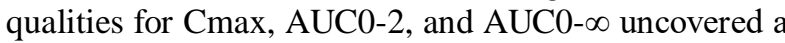
rough $2.7,2.8$, and 2.4 individual crease increments when rosuvastatin was given under fasted conditions contrasted with a bolstered organization. Be that as it may, because of enormous between mouse varieties, no noteworthy contrasts were found in Cmax, AUC0-2, or AUC0- $\infty$ among sustained and fasted mice (Table-1). These outcomes would recommend that nourishment doesn't essentially influence the oral pharmacokinetics of rosuvastatin in this mouse model; nonetheless, it is conceivable that enormous between mouse fluctuation is veiling a fundamental impact.

The liver-to-plasma fixation proportion of a medicine is a delicate marker of its hepatic take-up. In this way, to explore hepatic vehicle of rosuvastatin because of nourishment admission, we estimated the terminal plasma focus, liver fixation, and liver-toplasma fixation proportion of rosuvastatin in our sustained and fasted mice. At the terminal time point (2 hours), the normal plasma rosuvastatin focus was essentially more noteworthy for fasted mice when contrasted with encouraged mice (Figure-2A). In any case, liver groupings of rosuvastatin were not essentially unique among encouraged and fasted mice (Figure-2B). Mean liver-to-plasma focus proportion of rosuvastatin was 2.1-overlap more prominent in nourished mice when contrasted with fasted mice, anyway these qualities were not altogether unique (Figure-2C). Taken together, these outcomes propose that in this mouse model, hepatic take-up of rosuvastatin isn't changed by the nearness of nourishment. Notwithstanding, higher 2 hour plasma focuses in fasted mice and an inexact 2-overlap increment in liver-to-plasma fixation proportion in nourished mice when contrasted with fasted mice demonstrates that an impact might be conceal by it. 


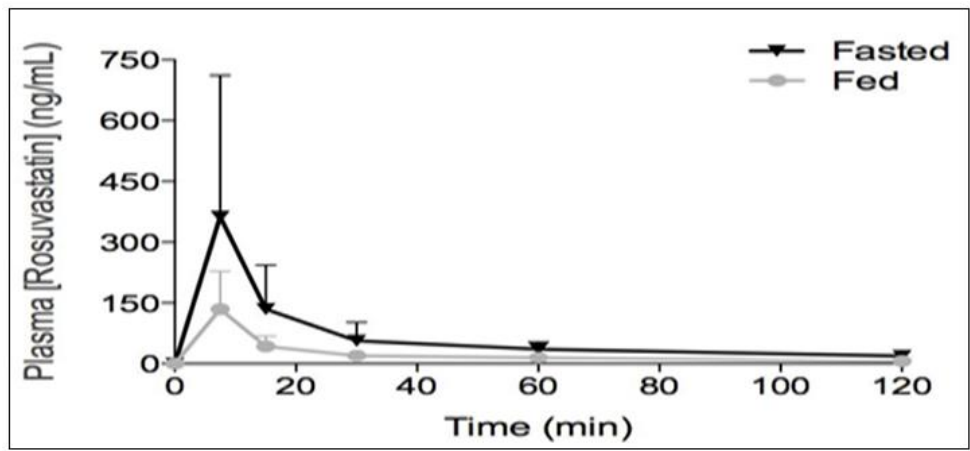

Fig-1: Plasma focus time bends of rosuvastatin in bolstered and fasted mice after organization of rosuvastatin by means of oral gavage

Plasma was gathered from blood tests taken at

$7.5,15,30,60$, and $120 \mathrm{~min}$ post portion. Plasma rosuvastatin fixations were estimated by LC-MS/MS. Information are introduced as mean $+\mathrm{SD}$.

Table-1: Analysis of pharmacokinetic parameters in fed and fasted wild-type mice after administration of rosuvastatin via oral gavage

\begin{tabular}{|l|l|l|l|}
\hline Parameter & Fasting $(\mathbf{n}=5)$ & Fed $(\mathbf{n}=4)$ & $\boldsymbol{p}$ \\
\hline $\mathrm{T}_{1 / 2}(\mathrm{~min})$ & $46.0(19.3)$ & $87.1(72.3)$ & 0.612 \\
\hline $\mathrm{C}_{\max }(\mathrm{ng} / \mathrm{mL})$ & $359.1(332.0)$ & $129.1(90.3)$ & 0.2993 \\
\hline $\mathrm{AUC}_{0-2}(\mathrm{ng} / \mathrm{mL})$ & $6714.4(4441.5)$ & $2221.1(1320.6)$ & 0.0421 \\
\hline $\mathrm{AUC}_{0-\infty}(\mathrm{ng} / \mathrm{mL})$ & $9301.2(5990.1)$ & $3432.1(1211.1)$ & 0.0512 \\
\hline
\end{tabular}

Data are presented as mean (SD)

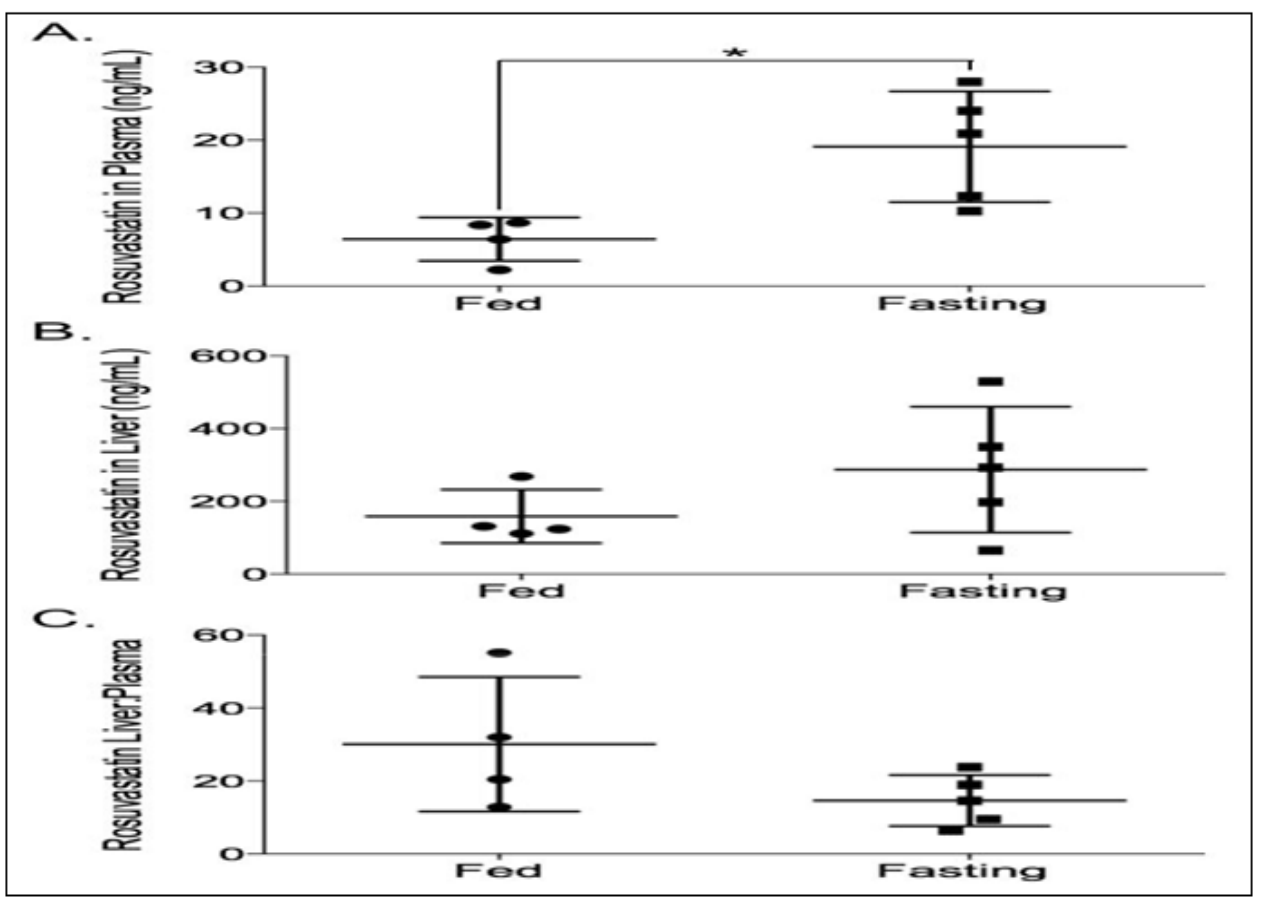

Fig 2(A, B, C)

Plasma was gathered from blood tests taken at 2 hours post portion. Livers were extracted 2 hours post portion and later homogenized. Rosuvastatin focuses in the plasma and liver homogenate tests were estimated by LC-MS/MS. Information introduced as mean with SD. Centrality of the mean contrast among sustained and fasted mice is portrayed by $* \mathrm{p}<0.05$.

\section{DISCUSSION}

Statin prescriptions are first-line pharmacotherapy for the treatment of hypercholesterolemia and anticipation of cardiovascular illness [7]. High flowing degrees of statins have been related with various statin-incited unfriendly occasions [8]. Critical proof shows that plasma levels of generally recommended statins, for example, pravastatin and atorvastatin, are lower when managed with nourishment 
contrasted with a fasted organization [9]. Study performed in solid Chinese subjects showed a significant decline in rosuvastatin plasma levels when rosuvastatin was directed with nourishment. We watched impressively lower AUC esteems when 10 $\mathrm{mg} / \mathrm{kg}$ rosuvastatin was regulated orally to bolster mice when contrasted with fasted mice. Nonetheless, to some extent because of test size and checked variety between mice, this distinction was not seen as critical.

Like Li et al., [10], we watched the general impact of diminished rosuvastatin introduction when it is taken with nourishment contrasted with organization without nourishment. Li et al., [10] presumed that rosuvastatin fundamental presentation was likely lower when controlled with nourishment because of weakened intestinal assimilation. In any case, lower foundational introduction could likewise be clarified by expanded hepatic take-up and leeway of rosuvastatin within the sight of nourishment. To research the commitment of hepatic take-up of rosuvastatin within the sight of nourishment, we controlled $10 \mathrm{mg} / \mathrm{kg}$ of rosuvastatin to encouraged and fasted mice and afterward looked at their plasma and liver medication focuses 2 hours post portion. We watched altogether higher plasma rosuvastatin focuses at the terminal time point in fasted mice when contrasted with feed mice. In addition, we watched no noteworthy distinction in the liver centralizations of rosuvastatin between the fed and fasted mice. The liver-to-plasma focus proportion of a medicine is a delicate marker of its hepatic take-up. Inside mice, mean liver-to-plasma fixation proportion was around 2.1-overlay higher in encouraged mice when contrasted with fasted mice managed oral rosuvastatin, despite the fact that this distinction was not critical. We watched rather enormous between mouse inconstancies in rosuvastatin levels and can't affirm contrasts in hepatic take-up of rosuvastatin when it is managed to sustained and fasted mice.

Baek et al., [5] found that Cmax and AUC of rosuvastatin were altogether lower when a $10 \mathrm{mg}$ oral portion was directed to hounds encouraged a high-fat feast when contrasted with those bolstered a low-fat dinner before dosing. Results from the present work demonstrate that taking rosuvastatin with nourishment brings about lower rosuvastatin fundamental presentation yet doesn't almost certainly influence its remedial activities.

\section{CONCLUSION}

In this examination we explored a potential nourishment impact on the plasma and liver degrees of rosuvastatin inside wild-type mice. This examination gives knowledge into the impact of nourishment on the oral pharmacokinetics of rosuvastatin. Our discoveries bolster our past theory that taking rosuvastatin with nourishment brings about lower coursing statin levels.
Here we exhibit that taking rosuvastatin with nourishment brings about lower fundamental introduction without trading off its remedial advantage. Along these lines, our discoveries can possibly fill in as a novel and basic methodology for relieving statin myopathy chance.

\section{REFERENCES}

1. Law, M. R., Wald, N. J., \& Rudnicka, A. R. (2003). Quantifying effect of statins on low density lipoprotein cholesterol, ischaemic heart disease, and stroke: systematic review and meta-analysis. Bmj, 326(7404), 1423.

2. Schachter, M. (2005). Chemical, pharmacokinetic and pharmacodynamic properties of statins: an update. Fundamental \& Clinical Pharmacology, 19(1), 117-125.

3. Gazzerro, P., Proto, M. C., Gangemi, G., Malfitano, A. M., Ciaglia, E., Pisanti, S., \& Bifulco, M. (2012). Pharmacological actions of statins: a critical appraisal in the management of cancer. Pharmacological Reviews, 64(1), 102-146.

4. Barakat, L., Jayyousi, A., Bener, A., Zuby, B., \& Zirie, M. (2013). Comparison of efficacy and safety of rosuvastatin, atorvastatin and pravastatin among dyslipidemic diabetic patients. ISRN Pharmacology, 2013.

5. Baek, I. H., Kwon, K. I., \& Kim, M. S. (2013). Effect of food on the pharmacokinetics of rosuvastatin after administration of a single oral dose in dogs. Drug Research, 63(3), 145-149.

6. Knauer, M. J. (2012). The Role of Drug Transporters in Statin-Induced Myopathy. Electronic Thesis and Dissertation Repository. Paper 1001.

7. Pencina, M. J., Navar-Boggan, A. M., D'Agostino Sr, R. B., Williams, K., Neely, B., Sniderman, A. D., \& Peterson, E. D. (2014). Application of new cholesterol guidelines to a population-based sample. New England Journal of Medicine, 370(15), 1422-1431.

8. Golomb, B. A., \& Evans, M. A. (2008). Statin adverse effects. American Journal of Cardiovascular Drugs, 8(6), 373-418.

9. Radulovic, L. L., Cilla, D. D., Posvar, E. L., Sedman, A. J., \& Whitfield, L. R. (1995). Effect of Food on the Bioavailability of Atorvastatin, an HMG"CoA Reductase Inhibitor. The Journal of Clinical Pharmacology, 35(10), 990-994.

10. Li, Y., Jiang, X., Lan, K., \& Jiang, Q. (2009). Comparative single- and multiple-dose pharmacokinetics of rosuvastatin following oral administration in Chinese volunteers. European Journal of Drug Metabolism and Pharmacokinetics, 34(3-4), 221-227. 